\title{
Article
}

\section{Gene Expression Profiles Reveal the Potential Link between Parkinson's Disease and Gastric Cancer}

\author{
Lei Zhang ${ }^{1}$, Zhiming $\mathrm{Ma}^{2^{*}}$, Suyan $\operatorname{Tian}^{3 *}$ \\ ${ }^{1}$ Department of Neurology, The Second Hospital of Jilin University, 218 Ziqiang Road, Changchun, Jilin, 130041, P.R. China \\ ${ }^{2}$ Department of Gastrointestinal Nutrition and Hernia Surgery, The Second Hospital of Jilin University, 218 Ziqiang Road, \\ Changchun, Jilin, 130041, P.R. China \\ ${ }^{3}$ Division of Clinical Research, The First Hospital of Jilin University, 71Xinmin Street, Changchun, Jilin, 130021, P.R. China
}

*Corresponding authors: Suyan Tian, E-mail: wxmt@jlu.edu.cn; Zhiming Ma,

E-mail: mazhiming@jlu.edu.cn

DOI: $10.37155 / 2717-5278-2021-03-02-2$

\begin{abstract}
Introduction: Gastric cancer (GC) is a cancer that develops from the lining of the stomach. Parkinson's disease (PD) is a long-term degenerative disorder of the central nervous system that mainly affects the motor system. Even though these two diseases seem to be distinct from each other, increasing evidences suggest that they might be linked. The objective of this study is two-fold. One is to explore if these two diseases are associated from the perspective of gene expression profiles. The other is to examine if race plays an interaction role on the association between these two diseases. Methods: Moderated t-tests or moderated paired t-tests were carried out to identify differentially expressed genes (DEGs) between the diseased people and the normal controls. Upon the overlapped DEGs between PD and GC, biological relevance was investigated using GeneCards database. Results and Discussion: Our analysis results showed that PD and GC are related to each other. Furthermore, our analysis suggest that these two diseases may be associated negatively with each other, and race is unlikely to play an interaction role on this association. Conclusions: This study may serve as a pilot study, and it will stimulate more work to investigate the link between distinct diseases using big omics data.

Keywords:Gastric cancer; Parkinson's disease; Differentially expressed genes (DEGs); Association; Gene expression profiles
\end{abstract}

\section{Introduction}

Gastric cancer (GC), also known as stomach cancer, is a cancer that develops from the lining of the stomach. In 2018, there were more than 1 million new cases of gastric cancer, with an estimated 783,000 deaths. One out of 12 deaths worldwide died of GC, making it the fifth most common cancer and the third leading cause of cancer deaths ${ }^{[1]}$. Particularly, it exerts a serious health problem in Eastern Asia. Parkinson's disease (PD) is a long-term degenerative disorder of the 
central nervous system that mainly affects the motor system. It is currently the second most prevalence neurodegenerative disorder after Alzheimer's disease and the most common movement disorder ${ }^{[2]}$. The causes of PD and GC are very complicated, and increasing evidences support the involvement of both genetic and environmental factors for both diseases.

These two diseases seem distinct from each other. Nevertheless, increasing evidence has indicated that they might be related. For example, it is believed that the gastrointestinal (GI) tract itself exists an intrinsic nervous system-the enteric nervous system (ENS). There are bidirectional interactions between the ENS and the Central nervous system (CNS) ${ }^{[3]}$. It is named as "brain-gut axis" ${ }^{[4]}$ and comprised of neural pathways, such as CNS, autonomic nervous system and hypothalamic-pituitary-adrenal axis. In the clinics, it was observed that a high proportion of PD patients suffer from constipation, abdominal distension and other GI symptoms before motor symptoms ${ }^{[5]}$. Therefore, we speculate that there might be a link between PD and GC.

Furthermore, several epidemiological studies ${ }^{[6-10]}$ have suggested that patients with $\mathrm{PD}$ had a reduced risk of having cancers including GC, compared to those without PD. Nevertheless, few studies have shown that the association between PD and cancers is positive, that means an increased risk of cancers for PD patients instead of a decreased risk ${ }^{[11,12]}$. For example, Lin's study ${ }^{[12]}$ showed that specifically for GC, the hazard ratio (HR) was estimated as 1.59 (95\% CIs: 1.30-1.94), meaning the risk of developing GC increased for PD patients. Also, the authors gave some explanation on this inconsistency and attributed it to that most of those epidemiological studies were carried out upon the Western population, in contrast their study population was the people in Taiwan island. Based on this, Lin et al. ${ }^{[12]}$ concluded that the race or/and environmental exposures have an interaction effect on the association between cancer and PD.

At the molecular level, several studies suggest that PD and cancers (including lung cancer, HCC, breast cancer and so on) are linked to each other with respects to somatic mutations, gene or miRNA expression.
Specifically for GC, one study ${ }^{[13]}$ showed that a specific miRNA, i.e., miR-148a not only was a potential tumor suppressor that inhibits gastric caner metastasis but also involved in neurological development and functions. Namely, the expression levels of miR-148a are lower in patients with PD compared to normal controls. Therefore, it is possible to investigate the connection between these two diseases from the level of molecular as well.

Microarray and RNA-seq technologies enable to monitor expression changes for thousands of genes simultaneously. For both GC and PD, numerous microarray experiments such as ${ }^{[14-20]}$ had already been conducted, with different objectives such as describing the expression difference between the diseased patients and normal controls and investigating the progress of the diseases. To the best of our knowledge, however, no study has been carried out to explore the link between GC and PD using gene expression profiles generated by high-throughput technologies. In this study, we intend to fulfill this gap to explore the potential association between these two diseases using gene expression data for GC and PD. Furthermore, separate analyses stratified by race for the GC cohort were also carried out to investigate the roles of race may play on the association between GC and PD.

\section{Methods and Materials}

\subsection{Experimental Data}

We searched the NIH's GEO (Gene expression omnibus) repository for the potential microarray experiments included in this study. First, the search for relevant gastric cancer data was conducted. The keywords used are gastric cancer, stomach cancer, human (before Dec, 2017). Then we restricted the platform to Affymetrix HG-U133 Plus 2.0 (GPL570) in addition to the availability of raw data (thus the preprocessing using the fRMA method is possible). The accession numbers of datasets included in this study are GSE13911 ${ }^{[18]}$, GSE19826 $6^{[19]}$ and GSE79973 ${ }^{[20]}$. In GSE79973 experiment, 10 paired GC and adjacent non-tumor tissues were included. The GSE19826 experiment collects 12 pairs of tumor tissues and their adjacent non-tumor tissues from GC patients, in addition to normal stomach tissues from three health people. The GSE13911 experiment was conducted in 
the area around Florence (Italy), and includes pairs of primary gastric tumors and their adjacent normal tissues.

Using the same inclusion criteria, the search for relevant data of PD was carried out, and it was found that only one experiment was eligible, i.e., GSE7621 ${ }^{[21]}$. The characteristics of these experiments including sample size, normalization method used and race are summarized in Table 1.

Table 1. Characteristics of microarray experiments in this study

\begin{tabular}{|c|c|c|c|c|c|c|c|}
\hline & Reference & Raw Data & Platform & $\begin{array}{c}\text { Normalization } \\
\text { (originally used) }\end{array}$ & $\begin{array}{c}\text { Diseased } \\
\text { people }\end{array}$ & Controls & Race \\
\hline \multicolumn{7}{|c|}{ Parkinson's disease } \\
\hline GSE7621 & {$[21]$} & Yes & GPL570 & MAS5.0 & 16 & \multicolumn{2}{c|}{ Hesperian (IT) } \\
\hline GSE13911 & {$[18]$} & Yes & GPL570 & MAS5.0 & $38(31)$ & 31 & Hesperian (IT) \\
\hline GSE19826 & {$[19]$} & Yes & GPL570 & MAS5.0 & 12 & $15(12)$ & Asian (CH) \\
\hline GSE79973 & {$[20]$} & Yes & GPL570 & MAS5.0 & 10 & 10 & Asian (CH) \\
\hline
\end{tabular}
cohort.

* Only paired normal tissues were used for gastric cancer (GC) datasets. IT: the Italian GC cohort; CH: the Chinese GC

\subsection{Pre-processing Procedures}

As shown in Table 1, same pre-processing method, i.e., MAS 5.0 was originally used among these four datasets to pre-process the raw data. In order to eliminate the batch effects from different experiments, however, raw data (CEL files) of the microarray data sets were downloaded from the GEO repository and pre-processed using the fRMA algorithm ${ }^{[22]}$, given the fRMA algorithm has been shown to provide an effective control on batch effects and is able to preprocess a single chip ${ }^{[22-24]}$. When there are multiple probe sets matched to one specific gene, the one with the largest absolute log fold change was used.

\section{Statistical Analysis}

\subsection{Differentially Expressed Genes (DEGs)}

Moderated t-tests using limma ${ }^{[25]}$ were carried out to identify differentially expressed genes (DEGs) between the PD patients and the PD-free subjects in the microarray data set, and those non-DEGs with an adjusted p-value/ a false discovery rate (FDR) using $\mathrm{BH}$ procedure to adjust for multiple comparison issue of $>0.05$ were filtered out. Since non-tumor tissues were from the same GC patients, the moderated paired t-tests were used to identify DEGs between the tumor tissues and the adjacent non-tumor tissues for the GC patients. The same value of 0.05 for FDR was used as the cutoff to determine if a gene is statistically significant. Lastly, the GC data were divided into two strata according to the race (or countries where the experiments were conducted), namely, GSE79973 and GSE19826 as one group and GSE13911 as the other group. They are referred to as the Chinese cohort $(\mathrm{CN})$ and the Italian cohort (IT) herein. There were 22-paired tissues in the CN cohort and 31 pairs in the IT cohort. The moderated paired t-tests were carried out again to identify DEGs on both cohorts.

\subsection{Protein-to-protein Interaction Network}

The String software ${ }^{[26]}$ was used to find the enriched pathways and to retrieve protein-to-protein interaction (PPI) information of the genes under consideration, upon the retreated PPI information the Cytoscape software ${ }^{[27]}$ was used to draw the network graph in which the isolated genes were omitted.

\subsection{Biological Relevance}

The Genecards database ${ }^{[28]}$ was used to investigate the biological relevance of identified DEGs for GC and PD. In this article, only directly associated genes with the phenotype of interest (either GC/stomach cancer or PD) are considered.

\section{Statistical Language and Packages}

All statistical analysis was carried out in the $\mathrm{R}$ language version 3.3 (www.r-project.org).

\section{Results and Discussion}

\subsection{Differentially Expressed Genes (DEGs)}

For GC, moderated paired t-tests identified 10,977 
differentially expressed genes (DEGs) between tumor tissues and their adjacent normal tissues. Two reasons may explain this genomic storm, referring to the resulting number of DEGs is large. First, the paired tumor and non-tumor tissues were used. Second, the sample size of this integrated data is sufficient enough to detect small difference. For PD, there were 441 DEGs between the diseased people and the normal controls. Among them, 248 genes overlapped with the DEGs of GC.

Separate analyses were carried out to investigate the association between GC and PD, stratified by ethnicity. For the $\mathrm{CN}$ cohort (including two experiments of GSE79973 and GSE19826), moderated t-tests identify 6,408 DEGs, among those genes there are 2,902 over-expressed genes and 3,506 down-expressed genes in the disease group, respectively. For the IT cohort, there are 8,724 DEGs, among which 3,634 genes are over-expressed and 5,090 are down-expressed. Venndiagrams (Figure 1) were presented to illustrate how the DEGs identified in the CN cohort, the IT cohort, and the integrated GC cohort (which combined both $\mathrm{CN}$ and IT cohorts together) are overlapped. Overall, there are 1,865 up-regulated genes and 2,846 downregulated commonly identified by both cohorts and the integrated GC dataset, making the overlapped number of DEGs to 4,711 .
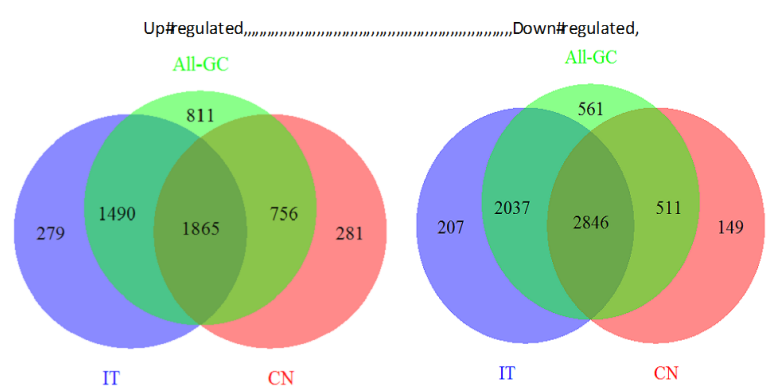

Figure 1. Venn-diagrams for differentially expressed genes identified by the gastric cancer Chinese cohort, the GC Italian cohort and two cohorts combined.

A. For the over-expressed genes using the adjacent non-tumor tissues as the reference group. B. For the down-expressed genes using the adjacent nontumor tissues as the reference. From these plots, it is observed that there are 1,865 up-regulated genes and 2,846 down-regulated commonly identified by both cohorts and the integrated GC dataset, making the overlapped number of DEGs to 4,711. CN: the Chinese cohort; IT: the Italian cohort.

\subsection{Biological Relevance}

Upon these 248 overlapped genes, their biological relevance with GC and PD was investigated by searching for the GeneCards database ${ }^{[28]}$. According to the Genecards database, 69 out of 248 overlapped DEGs are indicated to be relevant to GC. The number of biologically relevant genes to PD (with supports from previous studies as indicated by the GeneCards database) is 77 , of which 29 genes are also relevant to GC, making the overlapping proportion of the biologically meaningful DEGs between GC and PD to be $24.8 \%$ (Fisher's exact test: $\mathrm{p}=0.023$ ).

We further investigated those overlapped 29 genes indicated to be biologically relevant to these two diseases. First, for the 29 genes the directions of association for these two diseases were examined to investigate whether race exhibits an interaction effect on the relationship between PD and GC. Table 2 lists these 29 genes and their estimated association magnitudes/directions with PD and GC, respectively. From this table, it is observed that 8 out of 29 genes, namely, YY1, NUP93, CUX1, INSR, FKBP15, PDCD2, LUC7L2, and PEMT have the same association directions (i.e., either up-regulated or down-regulated in both diseases compared to their corresponding controls). The rest genes present opposite expression change directions for the comparisons of PD versus normal and of GC versus normal, implying when a patient has one of these two diseases, the risk of having the other disease may reduce. It supports the results given by most epidemiology studies conducted in the Western counties.

Table 2. Overlapped DEGs both directly associated with gastric cancer and Parkinson's disease

\begin{tabular}{|c|c|c|c|c|}
\hline Gene & $\begin{array}{c}\text { Estimate } \\
(\mathrm{PD})\end{array}$ & $\begin{array}{c}\text { Estimate } \\
(\mathrm{GC})\end{array}$ & Association & $\begin{array}{c}\mathrm{H} / \mathrm{A} \text { for } \\
\mathrm{GC}\end{array}$ \\
\hline HDGF & 0.3404 & -0.6418 & $\mathrm{~N}$ & $\mathrm{~A}$ \\
\hline YY1 & 0.5607 & 0.1363 & $\mathrm{P}$ & $\mathrm{H}$ \\
\hline HNRNPH3 & 0.5995 & -0.3442 & $\mathrm{~N}$ & $\mathrm{HA}(\mathrm{Y})$ \\
\hline KAT8 & 0.3153 & -0.1822 & $\mathrm{~N}$ & $\mathrm{H}$ \\
\hline DCC & -0.9907 & 0.111 & $\mathrm{~N}$ & $\mathrm{~A}$ \\
\hline
\end{tabular}


Continuation Table:

\begin{tabular}{|c|c|c|c|c|}
\hline Gene & $\begin{array}{l}\text { Estimate } \\
\text { (PD) }\end{array}$ & $\begin{array}{c}\text { Estimate } \\
(\mathrm{GC})\end{array}$ & Association & $\begin{array}{c}\mathrm{H} / \mathrm{A} \text { for } \\
\text { GC }\end{array}$ \\
\hline PEAK1 & 0.3911 & -0.2686 & $\mathrm{~N}$ & $\mathrm{HA}(\mathrm{Y})$ \\
\hline DIDO1 & 0.7418 & -0.2411 & $\mathrm{~N}$ & $\mathrm{H}$ \\
\hline NUP93 & -1.2527 & -0.3821 & $\mathrm{P}$ & $\mathrm{HA}(\mathrm{Y})$ \\
\hline SRSF1 & 0.4028 & -0.5703 & $\mathrm{~N}$ & $\mathrm{HA}(\mathrm{Y})$ \\
\hline SETD2 & 0.5539 & -0.1234 & $\mathrm{~N}$ & $\mathrm{H}$ \\
\hline CUX1 & 0.5925 & 0.1642 & $\mathrm{P}$ & A \\
\hline TRIM28 & 0.4229 & -0.7684 & $\mathrm{~N}$ & $\mathrm{HA}(\mathrm{Y})$ \\
\hline INSR & 0.8451 & 0.2365 & $\mathrm{P}$ & $\mathrm{HA}(\mathrm{Y})$ \\
\hline PTBP1 & 0.6683 & -0.5009 & $\mathrm{~N}$ & $\mathrm{HA}(\mathrm{Y})$ \\
\hline FKBP15 & 0.427 & 0.125 & $\mathrm{P}$ & $\mathrm{H}$ \\
\hline CIZ1 & 0.4691 & -0.283 & $\mathrm{~N}$ & $\mathrm{H}$ \\
\hline IGF1R & 0.6593 & -0.4338 & $\mathrm{~N}$ & $\mathrm{H}$ \\
\hline PDCD2 & -0.742 & -0.5376 & $\mathrm{P}$ & $\mathrm{HA}(\mathrm{Y})$ \\
\hline LUC7L2 & -0.6836 & -0.2748 & $\mathrm{P}$ & $\mathrm{H}$ \\
\hline HNRNPC & 0.4591 & -0.5953 & $\mathrm{~N}$ & $\mathrm{H}$ \\
\hline PEMT & -0.3997 & -0.3377 & $\mathrm{P}$ & $\mathrm{H}$ \\
\hline ALDH1A1 & -2.0877 & 1.7455 & $\mathrm{~N}$ & $\mathrm{HA}(\mathrm{Y})$ \\
\hline CREBBP & 0.7212 & -0.3476 & $\mathrm{~N}$ & $\mathrm{H}$ \\
\hline ABHD11 & -0.446 & 0.2273 & $\mathrm{~N}$ & $\mathrm{HA}(\mathrm{Y})$ \\
\hline GSTZ1 & -0.6303 & 0.3024 & $\mathrm{~N}$ & $\mathrm{H}$ \\
\hline KAT2A & 0.6963 & -0.5212 & $\mathrm{~N}$ & HA(Y) \\
\hline MCM7 & 0.5938 & -0.8929 & $\mathrm{~N}$ & $\mathrm{HA}(\mathrm{Y})$ \\
\hline FLCN & 1.0058 & -0.2902 & $\mathrm{~N}$ & $\mathrm{~A}$ \\
\hline STIP1 & 0.735 & -0.6953 & $\mathrm{~N}$ & $\mathrm{HA}(\mathrm{Y})$ \\
\hline
\end{tabular}

Note: $\mathrm{N}$ : the gene expression change directions in both diseases are opposite, meaning this specific gene is downexpressed in one disease group and over-expressed in the

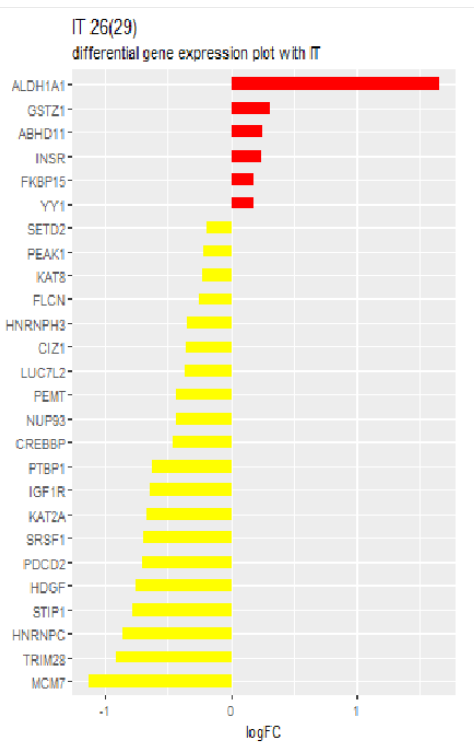

other disease. This may provide some evidence on both diseases are negatively correlated to each other; P: the gene expression change directions in both diseases are same, meaning this specific gene is either down-expressed in both disease groups or over-expressed in both disease groups. This may provide some evidence on that both diseases are postively correlated to each other. GC: gastric cancer; PD: Parkinson's disease. H: identified as DEGs by the Italian cohort; A: identified as DEGs by the Chinese cohort; (Y): the associated direction with gastric cancer is identical in both Italian and Chinese cohorts.

In order to testify that race may play an interaction role in the association between $\mathrm{PD}$ and $\mathrm{GC}^{[12]}$, indicators of whether the specific genes were identified by the $\mathrm{CN}$ cohort study and the IT study are given in Table 2. It is found that 13 out of 29 genes were identified by both cohorts and the combined GC study. Among these 13 genes, ten genes present opposite regulation directions when compared the association direction of GC with that of PD. This observation implies that despite of the race difference between $\mathrm{CN}$ cohort and IT cohort, their links to PD may be in consistent with each other, saying when a patient has one of these two diseases the risk of having the other disease decreases. In addition, a graph (Figure 2) was made to illustrate how the identified DEGs by separate $\mathrm{GC}$ cohorts were overlapped with these 29 genes, from which it was observed with a larger sample size and more uniform demographic characteristics, the IT cohort has a bigger overlapping proportion than the $\mathrm{CN}$ cohort does.

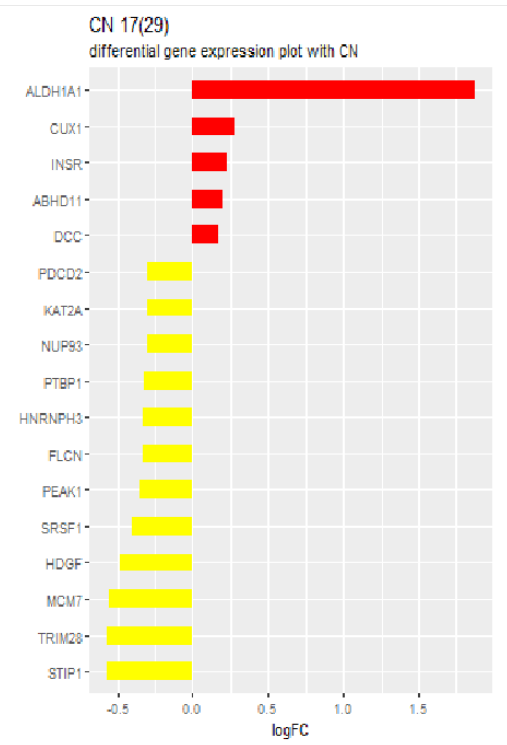

Figure 2. Overlaps between differentially expressed genes identified by two gastric cancer cohorts separately and the 29-gene list with biologically meaningful implications for both gastric cancer and Parkinson's disease. 
A. The Italian cohort. B. The Chinese cohort.

In these plots, red bars represent the corresponding genes were up-regulated in the GC group whereas yellow bars mean the corresponding genes were downregulated in the GC group. $\mathrm{CN}$ : the Chinese cohort. IT: the Italian cohort; PD: Parkinson's disease. GC: gastric cancer.

Heatmaps of normalized expression values (thus with a mean of 0 and a standard deviation of 1 ) for the 29-gene list were given separately for PD and
GC in Figure 3. In those plots, we found that a good separation between the PD patients and normal controls has been achieved using these 29 genes. In contrast, the separation between the GC tissues and their paired normal tissues is not so apparent. This may be due to that downsizing from more than 10,000 DEGs to the 29 overlapped DEGs with good biological implication excludes some genes with perfect discriminative capacity. Moreover, the remaining leftover of batch effects from several microarray studies may be another reason for the inferiority of separation between the GC tumor and non-tumor tissues.

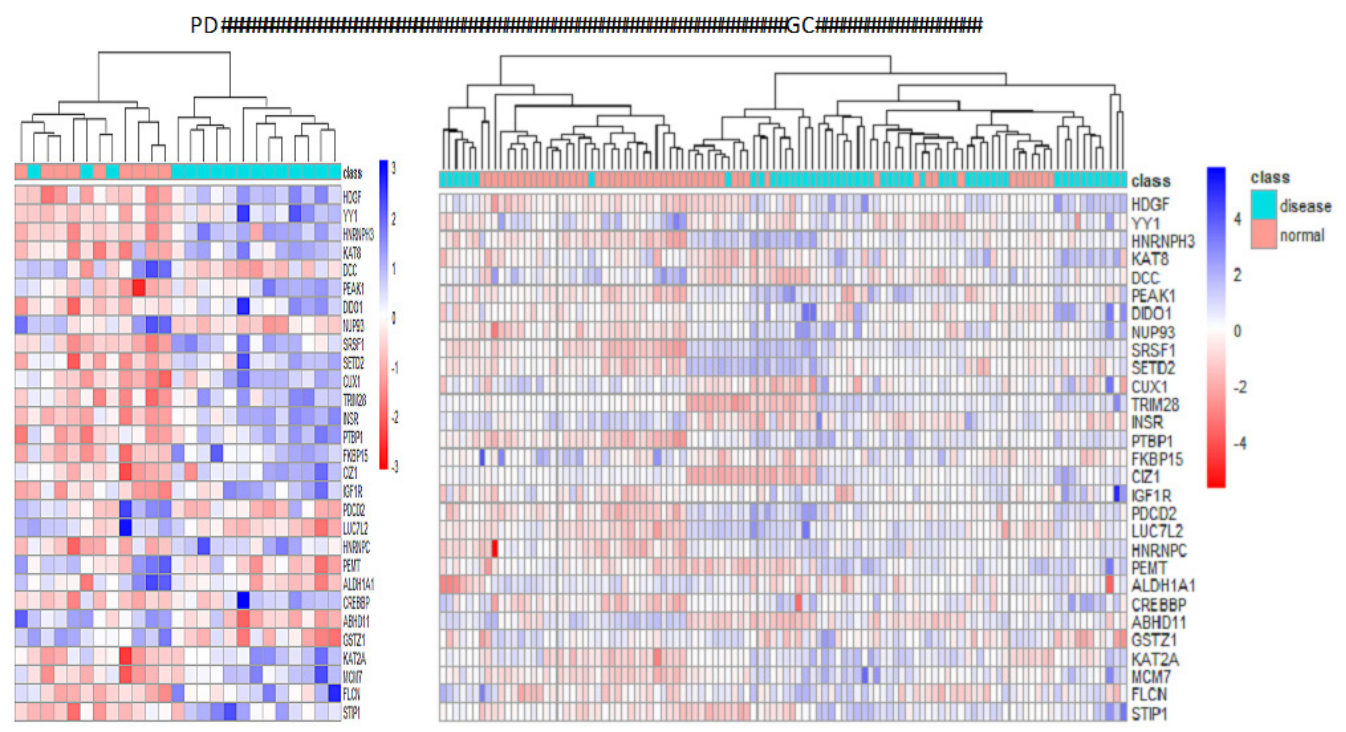

Figure 3. Heatmaps of identified 29-gene list. A. Parkinson's disease. B. Gastric cancer.

From these heat-maps, it is observed that a good separation between the PD patients and normal controls has been achieved using the overlapped 29 genes. In contrast, the separation between the GC tissues and their paired normal tissues is not so apparent. This may be due to that downsizing from more than 10,000 DEGs to the 29 overlapped DEGs with good biological implication excludes some genes with perfect discriminative capacity. PD: Parkinson's disease; GC: gastric cancer.

\subsection{On the Interaction Network Level}

Given the fact that the sample size of integrated $\mathrm{GC}$ dataset is about twice of those two individual cohorts (thus having a better statistical power to detect DEGs), both the network construction and the pathway enrichment analysis were carried out upon the DEGs identified by the integrated GC dataset.

Upon the protein-to-protein interaction (PPI) information of the overlapped 29 biologically relevant genes for both PD and GC given by the String software, the corresponding network was drawn using the Cytoscape software ${ }^{[27]}$ and is presented in Figure 4. In this graph, there are two big sub-networks and one small sub-network with a size of 2 . The isolated genes were omitted in the constructed network. Two copies of the constructed network were made, one for GC and the other for PD. On these two plots, the up-regulated genes and the down-regulated genes were highlighted in different colors. Obviously, the expression values of all except 5 genes change on the opposite directions, in which all hub genes within the two big sub-networks (i.e., KAT2A, CEBBP and SRSF1) were included. A 
hub gene is located in or near the center of a network and has high connectivity level, therefore, its subtle

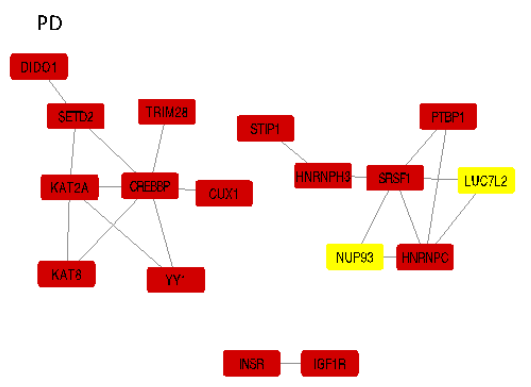

expression change is more likely to have a significant impact than a barely connected gene is.

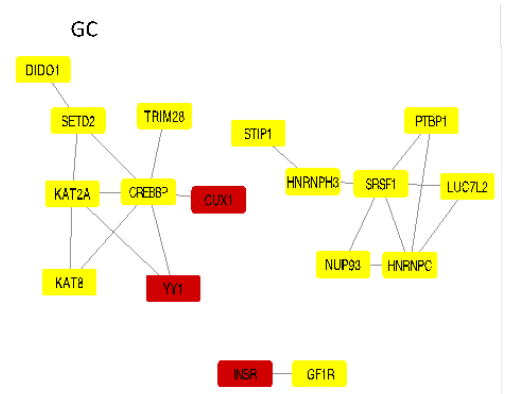

Figure 4. Gene-to-gene interaction network of the identified 29-gene list

On this network, the isolated genes were omitted. The network was constructed using the String database and Cytoscape software. Two copies of the constructed network were made, one for GC and the other for PD. On these two plots, the up-regulated genes and the down-regulated genes were highlighted in red and yellow, respectively. From these plots, it is observed that the expression values of all but 5 genes (i.e., YY1, CUX1, INSR, NUP93, and LUC7L2) change on the opposite directions, in which all hub genes within the two big sub-networks (i.e., KAT2A, CEBBP, and SRSF1) were included.

\subsection{Pathway Enrichment Analysis}

The String software ${ }^{[26]}$ was used to search for the enriched pathways by the 69 GC-related genes the 77 PD-related genes, in order to explore the function of the biologically meaningful overlapped DEGs between PD and GC. With regards to the GO biological process (BP) category, there are 33 terms enriched by GC-related genes and 43 terms enriched by PD-related genes. Among these terms, 7 were overlapped between the two disease-related gene lists. Twenty-two of chemical component (CC) terms are indicated as enriched pathways of GC-related genes, 17 are enriched pathways of PD-related genes and the number of overlapped terms are 11. In terms of GO molecular function (MF) and KEGG pathway, the numbers are 0 and 14 for GC-related genes, 3 and 1 for PD-related genes, respectively. No overlaps exist among either the GO MF terms or KEGG pathways. In Figure 5, the overlapped GO terms are presented along with their significance levels (i.e., false discovery rate of enrichment analysis) in both GC-related gene lists and PD-related gene lists. These terms deserve more future investigation, in order to decipher completely the links between PD and GC from the prospective of pathway level.

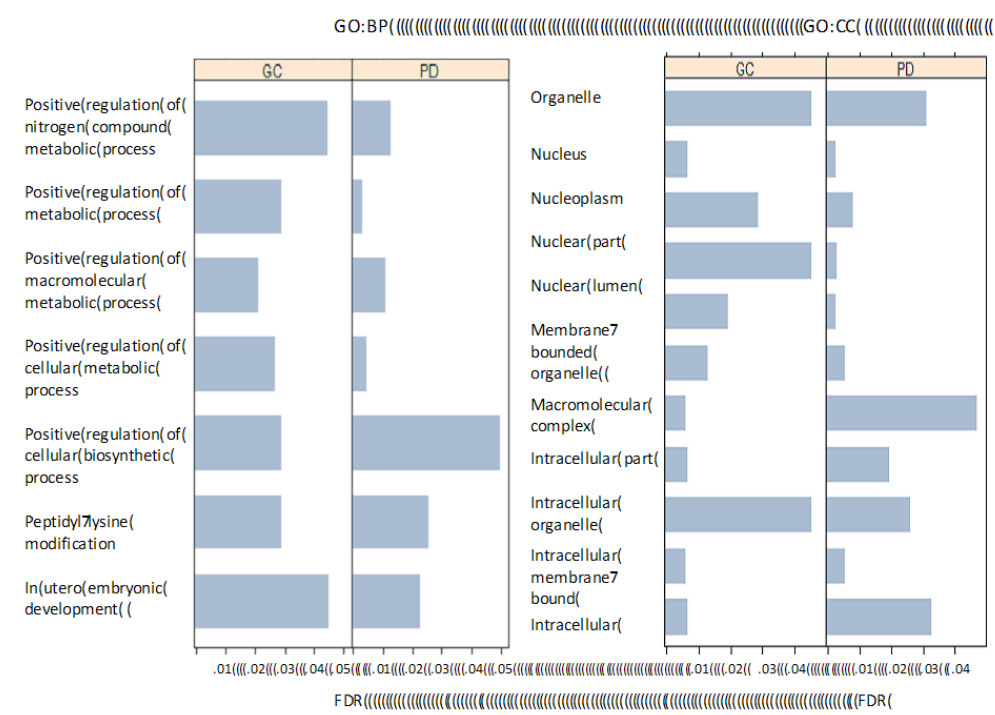

Figure 5. Overlapped gene ontology terms enriched by the identified PD-related genes and by the identified GC-related genes. PD: Parkinson's disease; GC: gastric cancer; BP: Biological Processes; CC: Cellular Components. 


\section{Conclusions}

Using gene expression profiles, it is found that two distinct diseases, namely, PD and GC are linked to each other. Nevertheless, this study has several limitations. First, more ideal study design is that among GC patients the status of PD would be checked or among PD patients the status of GC would be checked. More specifically, the association analysis should be carried out on the same set of patients as that in an epidemiology study. For example, among PD patients the baseline expression values between those developed cancer and those without cancer are compared to investigate the expression change directions. In reality, however, the corresponding clinical information such as the status for other malignancies other than the disease under investigation is usually unavailable so that the use of same cohorts to test the association between the two diseases is impossible.

On the other hand, there is no microarray experiment for PD on the Eastern Asian population. Our exclusive search on PD data only returned the studies that were carried out in Western countries, even without the chip platform restriction. Therefore, separate analyses on the same race to examine the association between PD and GC were impossible. Currently, we have obtained the direction of DEGs for the Hesperian PD patients and the Hesperian GC patients, and that of DEGs for the Hesperian PD patients and the Asian GC patients. These two directions are in consistent with each other, which shred some evidence on that race might play no interaction role on the association between PD and GC. Certainly, the possibility that the DEGs have different direction for the Asian PD patients cannot be excluded, even though we searched for the literature and found no support for it. Further investigation is definitely warranted.

Second, the sample sizes of these experiments are moderate and may not provide a good representative of the Hesperian/Asian population. However, the study design for the GC experiments is better than that for the PD study since for the GC studies the paired normal issues from the same patients were used. Therefore, this study has a better control over the potential confounding effects from the confounders such as age, gender and environment factors.
Lastly, when Lin's study ${ }^{[12]}$ explored the association between PD and GC, the survival time of GC patients is the outcome. In many applications, we observed that the prognostic gene signature for a specific disease is barely overlapped with the gene signature that differentiates the diseased patients apart from the normal controls. For the GC microarray data used in this study, the survival information for patients is missing, thus it is impossible to obtain the prognostic gene signature for GC. This study only shed some doubt on, but cannot invalidate completely the conclusion given by Lin's study ${ }^{[12]}$ since the outcomes of interest differ in these two studies.

In our future work, two questions worth of being further investigated and exclusively examined will be focused: the first question is about the association direction. While there is indeed a link between GC and PD given there are DEGs shared by the comparison of GC patients versus controls and that of PD patients versus controls, how are they linked - a positive or negative association? The second one is whether and how race or environmental factors affect this association. To conclude, this study may serve as a pilot study, and it will stimulate more research to investigate the relationship between GC and PD from the prospective of gene expression profiles.

\section{Availability of Data and Materials}

Microarray datasets were downloaded from the GEO database, all data are open and publicly available.

\section{Competing interests}

No competing interests have been declared.

\section{Funding}

This study was supported by a fund (JJKH20190032KJ) from the Education Department of Jilin Province and a fund (2019SRCJ022) from the Jilin Province Department of Finance.

\section{Acknowledgement}

Not applicable.

\section{References}

[1]. Bray F, Ferlay J, Soerjomataram I: Global Cancer 
Statistics 2018 : GLOBOCAN Estimates of Incidence and Mortality Worldwide for 36 Cancers in 185 Countries. CA: A Cancer Journal for Clinicians 2018:394-424.

[2]. Obeso JA, Stamelou M, Goetz CG, Poewe W, Lang AE, Weintraub D, Burn D, Halliday GM, Bezard E, Lehericy S, Brooks DJ, Rothwell JC, Delong MR, Marras C, Tanner CM, Ross GW, Langston JW, Klein C, Bonifati V, Jankovic J, Lozano AM, Deuschl G, Bergman H, Tolosa E, Fahn S, Postuma RB, Berg D, Marek K, Surmeier DJ, Olanow CW, Kordower JH, Stoessl AJ, Hospital A, Shulman G, Clinic MD, Hospital W, Illness M, J CM, Sciences M, Centre M, Biology C, Translational C, Initiative N: Past, Present, and Future of Parkinson's Disease: A Special Essay on the 200th Anniversary of the Shaking Palsy J.A. Movement Disorders 2017, 32:1264-1310.

[3]. Chalazonitis A, Rao M: Enteric nervous system manifestations of neurodegenerative disease. Brain Research 2018.

[4]. Bercik P, Collins S, Verdu E: Microbes and the gut-brain axis. Neurogastroenterology \& Motility 2012:405-413.

[5]. Malek N, Lawton MA, Grosset KA, Bajaj N, Barker RA, Burn DJ, Foltynie T, Hardy J, Morris HR, Williams NM, Ben-shlomo Y, Wood NW: Autonomic Dysfunction in Early Parkinson, s Disease : Results from the United Kingdom Tracking Parkinson's Study. Movement Disorders 2016:509-516.

[6]. Driver JA: Inverse association between cancer and neurodegenerative disease : review of the epidemiologic and biological evidence. Biogerontology 2014, 15:547-557.

[7]. Driver JA: Understanding the link between cancer and neurodegeneration. Journal of Geriatric Oncology 2011, 3:58-67.

[8]. Wirdefeldt K, Weibull CE, Chen H, Kamel F, Lundholm C, Fang F: Parkinson's Disease and Cancer : A Register-based Family Study. American Journal of Epidemiology 2014, 179:85-94.

[9]. Bajaj A, Driver JA, Schernhammer ES: Parkinson's disease and cancer risk : a systematic review and meta-analysis. Cancer Cause Control 2010, 21:697-707.

[10]. Lo RY, Tanner CM, Eeden SK Van Den, Albers
KB, Leimpeter AD, Nelson LM: Comorbid Cancer in Parkinson's Disease. Movement Disorders 2010, 25:1809-1817.

[11]. Elbaz A, Peterson BJ, Bower JH, Yang P, Maraganore DM, Mcdonnell SK, Ahlskog JE, Rocca WA: Risk of Cancer After the Diagnosis of Parkinson's Disease : A Historical Cohort Study. Movement Disorders 2005, 20:719-725.

[12]. Lin P-Y, Chang S-N, Hsiao T-H, Huang B-T, Lin C-H, Yang P-C: Association Between Parkinson Disease and Risk of Cancer in Taiwan. JAMA ONCOl 2015:1-8.

[13]. Hu C, Tseng C, Chien C, Huang H, Ku W: Quantitative Proteomics Reveals Diverse Roles of miR-148a from Gastric Cancer Progression to Neurological Development. Jounal of Proteome research 2013, 12:3993-4004.

[14]. Zheng B, Liao Z, Locascio JJ, Lesniak KA, Roderick SS, Watt ML, Eklund AC, Zhang-james Y, Kim PD, Hauser MA, Grünblatt E, Moran LB, Mandel SA, Riederer P, Miller RM, Federoff HJ, Wullner U, Parapetropoulos S, Youdim MB, Cantuti-Castelvetri I, Young AB, Vance JM, Davis RL, Hedreen JC, Adler CH, Beach TG, Graeber MB, Middleton FA, Rochet J-C, Scherzer CR, Consortium TGPGE: PGC-1 $\alpha$, A Potential Therapeutic Target for Early Intervention in Parkinson's Disease. Sci Tansl Med 2010, 2:52ra73.

[15]. Moran L, Duke D, Deprez M, Dexter D, Pearce R, Graeber M: Whole genome expression profiling of the medial and lateral substantia nigra in Parkinson 's disease. Neurogenetics 2006, 7:1-11.

[16]. Zhang Y, James M, Middleton FA, Davis RL: Transcriptional Analysis of Multiple Brain Regions in Parkinson's Disease Supports the Involvement of Specific Protein Processing, Energy Metabolism , and Signaling Pathways, and Suggests Novel Disease Mechanisms. American Journal of Medical Genetics Part B 2005, 137B:5-16.

[17]. Kim S, Kim J, Lee H, Noh S, Song K, Cho J, Jeong H, Kim WH, Yeom Y, Kim N, Kim S, Yoo H, Kim YS: Molecules and Meta- and Gene Set Analysis of Stomach Cancer Gene Expression Data. Molecules and Cells 2007, 24:200-209.

[18]. Errico MD, Rinaldis E De, Blasi MF, Viti V, Falchetti M, Calcagnile A, Sera F, Saieva C, 
Ottini L, Palli D, Palombo F, Giuliani A, Dogliotti E: Genome-wide expression profile of sporadic gastric cancers with microsatellite instability. European Journal of Cancer 2008, 45:461-469.

[19]. Wang Q, Wen Y-G, Li D-P, Xia J, Zhou C-Z, Yan D-W, Tang H-M, Peng Z-H: Upregulated INHBA expression is associated with poor survival in gastric cancer. Med Oncol 2012, 29:77-83.

[20]. He J, Jin Y, Yao H-B, Xia Y-J, Ma Y, Wang W, Shao Q-S: Downregulation of ALDOB is associated with poor prognosis of patients with gastric cancer. OncoTargets and Therapy 2016, 9:6099-6109.

[21]. Lesnick TG, Papapetropoulos S, Mash DC, Ffrench-mullen J, Shehadeh L, Andrade M De, Henley JR, Rocca WA, Ahlskog JE, Maraganore DM: A Genomic Pathway Approach to a Complex Disease : Axon Guidance and Parkinson Disease. PLoS Genetics 2007, 3:e98.

[22]. McCall MN, Bolstad BM, Irizarry RA: Frozen robust multiarray analysis (fRMA). Biostatistics 2010, 11:242-253.

[23]. McCall MN, Irizarry RA: Thawing Frozen Robust Multi-array Analysis (fRMA). BMC Bioinformatics 2011, 12:369.
[24]. McCall MN, Jaffee HA, Irizarry RA: fRMA ST: frozen robust multiarray analysis for Affymetrix Exon and Gene ST arrays. Bioinformatics 2012, 28:3153-4.

[25]. Smyth G: Limma: linear models for microarray data. In ... and computational biology solutions using $R$ and .... edited by R. Gentleman, V. Carey, S. Dudoit, R. Irizarry WH (eds. . New York: Springer; 2005:397-420.

[26]. Franceschini A, Szklarczyk D, Frankild S, Kuhn M, Simonovic M, Roth A, Lin J, Minguez P, Bork P, von Mering C, Jensen LJ: STRING v9.1: protein-protein interaction networks, with increased coverage and integration. Nucleic acids research 2013, 41:D808-15.

[27]. Smoot ME, Ono K, Ruscheinski J, Wang P-L, Ideker T: Cytoscape 2.8: new features for data integration and network visualization. Bioinformatics 2011, 27:431-2.

[28]. Safran M, Dalah I, Alexander J, Rosen N, Stein TI, Shmoish M, Nativ N, Bahir I, Doniger T, Krug H, Sirota-madi A, Olender T, Golan Y, Stelzer G, Harel A, Lancet D: GeneCards Version 3 : the human gene integrator. Database 2010, 2010:baq020. 